\title{
Therapeutic Strategies to Tackle Burnout and Emotional Exhaustion in Frontline Medical Staff: Narrative Review
}

\author{
Daniel C McFarland (D) \\ Fay Hlubocky ${ }^{2}$ \\ 'Department of Medicine, Northwell \\ Health Cancer Institute, Lenox Hill \\ Hospital, New York, NY, USA; \\ ${ }^{2}$ Department of Medicine, Section of \\ Hematology/Oncology, University of \\ Chicago, MacLean Center for Clinical \\ Medical Ethics, and the Cancer Research \\ Center, Chicago, IL, USA
}

\begin{abstract}
Burnout and emotional exhaustion in frontline healthcare workers and their implications for the health of patients, individual clinicians, and organizations are increasingly described among various healthcare settings. Yet therapeutic strategies to address burnout and other work-related conditions are outpaced by innumerable descriptions of burnout prevalence across healthcare disciplines. This review provides a framework that should be helpful in beginning the process of addressing burnout and its related conditions. It begins with an elucidation of key inter-related concepts of work-related conditions that should be considered in the differential diagnosis along with other mental health conditions that are concomitantly elevated in healthcare clinicians (eg, depression and substance abuse) but require a different approach to treatment. Factors that lead to increased resilience, engagement, and thriving in clinical workplaces are considered. While strategies are dichotomized between organizational level interventions and individual or personal interventions to address burnout, the idea of identifying and addressing root causes of burnout and related conditions is highlighted. The efficacy and feasibility of interventions that incorporate mindfulness-based stress reduction, cognitive behavioral strategies, meaning-centered therapy, and compassion training are highlighted as interventions with proven efficacy and durability that should be considered based on work-related stressors and appeal to clinicians.
\end{abstract} Keywords: burnout, clinician wellness, clinician well-being, emotional exhaustion, resilience, front-line staff

\section{Introduction}

\section{Hypothetical Case}

Mary is a 56-year-old family practice physician who is in disbelief that she is contemplating early retirement. Work has always been more than a job. It's a lifelong calling for her. But recently, she noticed waning satisfaction from patient care and is exhausted perpetually after several administrative changes at work requiring her to work longer hours and weekends. She feels unaccomplished in her practice and unappreciated by her colleagues. She is increasingly afraid of making mistakes and that her medical knowledge is not sufficient. She feels overwhelmed with electronic health record (EHR) that accompanies her home every night to be completed after dinner. She is uncharacteristically irritable and often filled with contempt for her colleagues and patients. She used to feel that she could help almost anyone but now feels that many of her patients are hopeless. Outside of work, she enjoys some activities but has not continued her exercise and some self-
Correspondence: Daniel C McFarland Department of Medicine, Northwell Health Cancer Institute, Lenox Hil Hospital, New York, Ny, USA

Tel + I 212-434-4460

Fax + I 646-227-7283

Email danielcurtismcfarland@gmail.com 
care. She feels hypocritical giving health-oriented advice that she is no longer following. She dreads going back to work at the beginning of each week. Close colleagues have notices changes in her attitude and demeanor, but her employer is not concerned since patients have not complained and believes that she will eventually snap out of it. Of note, this is a hypothetical case.

\section{Introduction}

This case highlights several typical issues with burnout and its related conditions. It is insidious and is often concealed by other factors (eg, maybe I am just getting older, patients/colleagues/practices are changing, it's just a phase). Early retirement is one of the many potential consequences of untreated burnout. While this solution seems appealing to many clinicians in the throes of burnout, its effect on the healthcare system is significant and does not change the underlying issues that caused burnout in the first place. Increasingly, organizations are invested in preventing and treating burnout because the fallout of burnout is not only poor quality of life for clinicians and reduced quality of patient care and medical errors but economic as clinicians leave practices and turnover is an increasingly expensive ordeal.

Aside from emotional exhaustion, Mary expresses a sense of decreased professional accomplishment, which is expressed in multiple ways from fear of medical errors, desire to retire, not keeping up with colleagues, poor selfesteem, loss of meaning in her career. Her case also highlights cynicism as she now looks at colleagues with scorn and sees her patients as hopeless. In a sense, her work is on autopilot with the joy having left long ago-this sense of depersonalization and cynicism is characteristic and a defining of burnout along with emotional exhaustion and sense of reduced personal accomplishment. Most notably, her sense of self is affected, and the long-term consequences are worrisome.

Mary's case is also highly concerning for other mental health conditions not directly related to work, but which would affect her work such as depression, substance use or chemical dependence, neurodegenerative or cognitive disorders, other affective or psychotic conditions, and risk of suicide. Unfortunately, mental health and substance abuse issues are higher in physicians along with risk of suicide. Her history reveals symptoms typical of burnout and its severity tied to her work environment. But, ruling out these other prevalent conditions would be prudent. While burnout and its symptoms mimic depression, they are tied to the work environment such that they remit once away from work or changes are made. Additionally, burnout should not cause anhedonia, a heightened sense of guilt, or suicidal thinking. Self-esteem is affected in relation to work but not universally.

This paper will discuss burnout and its related conditions in frontline medical staff highlighting therapeutic strategies that may be useful for psychologist to implement in addressing these issues.

\section{Key Concepts of Work-Related Mental Health Conditions}

Burnout is associated with several other work-related conditions such as compassion fatigue, and moral distress. Burnout should be distinguished from other work-related conditions since the approach to treatment ought to vary accordingly. Interestingly, each of these concepts, which describe pathological states or work-related dysfunction are countered by opposite concepts describing healthy work-related functioning. That is, addressing burnout should lead to engagement; addressing compassion fatigue should lead to compassion satisfaction; and addressing moral distress should lead to moral success. These later concepts should be incorporated into clinician-workplace wellness and can be thought of in terms of underlying clinician resilience. Where one concept is emphasized, the others are likely to follow and addressing one would likely result in improving each concept.

Burnout as a clinical condition was first described in the mid-1970s by psychologist Herbert Freudenberger, ${ }^{1}$ burnout is a condition that occurs when work stress coupled with additional life pressures exceed the ability to cope resulting in physical and mental distress. He described burnout as a "state of mental and physical exhaustion caused by one's professional life" seen predominately in human service workers. It is defined by its three component parts: emotional exhaustion, depersonalization (or cynicism, which is more common in males), and a depleted sense of professional accomplishment. Of these domains, the first two are the most important as depletion of professional accomplishment may be secondary to the other two domains.

The concept of physicians in distress is not new. Reports of poor coping, addiction, depression, and suicide existed even in the time Hippocrates. ${ }^{6,7}$ More recently, physicians' experience of burn-out syndrome-emotional exhaustion, negativism or cynicism towards one's job, 
and reduced professional efficiency as classified by the International Classification of Disease $11^{\text {th }}$ edition ${ }^{8}$-has been well described across all medical disciplines and is highly applicable to oncology, especially among younger oncologists. ${ }^{9}$ The burn-out syndrome describes a mismatch between worker and workplace and is distinct from depression although symptoms may overlap. ${ }^{10}$ Unfortunately, both are highly prevalent in oncology and lead to reduce work efficacy, medical errors, job dissatisfaction, and turnover. ${ }^{11,12}$

The Maslach Burnout Inventory (MBI) is the most widely used scale to measure burnout and incorporates those three domains. It was the first scale to measure burnout and was developed in $1981 .^{2}$ The Copenhagen Burnout Inventory (CBI) was developed in 2005 and consists of three scales measuring personal burnout, workrelated burnout, and client-related burnout that can be used in these different domains. ${ }^{3}$ Many of the symptoms of burnout mimic depression and some researchers posit that burnout is only secondary to underlying depression. But the key distinction is its relationship with the work environment such that changes in the work environment directly affect the presence or absence of burnout but do not affect depression. Burnout is synonymous with disengagement from work through its symptoms. For example, distancing occurs with emotional exhaustion, depersonalization (ie, treating clients as objects and not engaging with them), and a belief in not being effective disincentivizes more work activity. While burnout is inherently a mismatch between worker and workplace and any clinician may be vulnerable to it, there are several notable features associated with burnout. Rates of burnout are typically higher in clinicians who are female, single or without a partner, younger, eager to achieve, highly driven and ambitious, and those who are willing to cross boundaries (eg, allowing for work during personal time or to enter other life domains). In addition, burnout subtypes provide descriptive evidence of the syndrome and its multifaceted manifestations. ${ }^{4}$ While frenetic, underchallenged, and worn-out burnout subtypes have been described with validated scales, they have not been readily adapted into the common measurement and parlance of burnout.

Engagement is mutually exclusive with burnout and is the goal of burnout prevention or treatment measures. Engagement depends on alignment of work environment and personal attributes. It can be cultivated in clinicians' attitude, behaviors, motivations, and worldview. Clinicians who are engaged feel nourished by their work, have personal agency, and feel that they have the means to affect outcomes. There is a sense that one's work makes a difference. ${ }^{5}$ Conversely, engagement may come off balance when work is driven by fear, escapism, or compulsion, and the sense that one's work is of little benefit to anyone including oneself, which describes the bleak experience of burnout leading to fatigue, pessimism, cynicism, physical and mental illness. ${ }^{5}$ The experience of Mary in the case above highlights a first-hand account of how she become increasingly less engaged.

Burnout rates are consistently high across healthcare sectors. Surprisingly, medical fields most immediately involved with the stress of managing patients at the end of life have average burnout rates. Also, burnout is also high in fields that are not directly involved with first line patient care even in the midst of a global pandemic such as COVID-19. ${ }^{6}$ A recent comparison of physician burnout and satisfaction with work-life integration between 2011 and 2017 found that $43.9 \%$ had at least one symptom of burnout on the Maslach Burnout Inventory (MBI) in 2017 compared with $54.4 \%$ in 2014 and $45.5 \%$ in $2011 .{ }^{7}$ In addition, clinicians working in smaller organizations, working longer hours, being under 50 and working weekends were associated with burnout. ${ }^{8}$ These work-related and individual factors have greater relevance than the clinician's particular medical discipline even though specific disciplines engender unique stressors that may lead to burnout. For example, burnout in psychiatry has been linked to having experienced violence on the job (eg, battered by patient), which is surprisingly common. The high rates of burnout in emergency medicine (roughly $75 \%$ in 2014) are likely related to unpredictability of workload and structure. ${ }^{9}$

The implications of burnout are vast. It is associated with poor outcomes for patients, clinicians, and organizations. In addition to substandard medical care (not meeting standards in clinical practice), burnout is directly related to medical errors, and patient outcomes with treatments, quality, and safety. ${ }^{10}$ On a biological level, burnout in clinicians can lead to thinning of the pre-frontal cortex (PFC) mediated through uncontrollable stress. This finding is particularly troubling since the PFC governs many cognitive operations essential to physicians, including abstract reasoning, higher-order decision-making, insight, and the ability to persevere through challenges. ${ }^{11}$ The prefrontal cortex is reliant on arousal state and becomes impaired under conditions of fatigue or incontrollable stress. In short, reduced prefrontal cortex self-regulation may 
explain several challenges associated with burnout in physicians, including reduced motivation, unprofessional behavior, and suboptimal communication with patients. ${ }^{11}$

Compassion fatigue results from vicarious traumatization occurring through patients whose stories and clinical situations directly affect their professional caregivers. It has many similarities with post-traumatic stress disorder (PTSD) and is also called secondary traumatic stress. ${ }^{12}$ Compassion fatigue can be distinguished from burnout based on its immediate onset, even following a particularly challenging clinical encounter. Compassion fatigue is directly related to the clinical aspects of work and its vicarious traumatization. Burnout appears insidiously and may be related to multiple aspects of one's work or professional life. Whie the concept of compassion fatigue is contested by some experts, ${ }^{13}$ clinicians can and do experience vicarious traumatization related to the compassion aspect of clinical work. At the same time, these clinical experiences related to clinical connectedness may be experienced as meaningful or the related term, compassion satisfaction, which results from the rewarding feeling of caring for another and a sense of accomplishment. ${ }^{14}$ Compassion satisfaction has been defined as the pleasure derived from doing clinical work well. It stands in sharp contrast to compassion fatigue where work is a source of negativity for the clinician. Compassion satisfaction may result from the feeling that the clinician is able to provide a reflection of how they believe the world should be in terms of patient care, when one's work aligns with the clinician's belief system. ${ }^{6}$ A large Canadian study found that when clinicians were involved with multiple compassion requiring tasks they became more likely to report compassion fatigue and burnout, but no significant difference was seen in levels of compassion satisfaction. ${ }^{15}$ The study authors hypothesized that even staff who did not provide direct clinical care derived compassion satisfaction from their work.

Moral distress arises when the clinician is aware of a moral problem, can determine a remedy, but is unable to act on it because of internal or external constraints. ${ }^{6}$ This inability to act ethically makes the clinician feel compromised in their duties, usually imposed upon by an authority who is creating or perpetuating the source of moral distress. A Moral injury is a psychological wound resulting from witnessing or participating in a morally transgressive act and may be experienced cumulatively. ${ }^{5}$ They leave a "moral residue" that is cumulative and erodes the clinician's sense of wellbeing over time. ${ }^{16}$ On the other hand, moral success has been described when the work place encourages effective communication about morally or ethically charged situations that provides for beneficial outcomes and growth of clinicians in various professional ranks. "Ethical environments" are described where ethical concerns are freely shared, communicated, and investigated as a collective learning process that is an anecdote to moral distress. Moral success forms an important part of what has been described philosophically as selfcontentment (ie, Selbstzufriedenheit), which would certainly relate contentment in the work environment. ${ }^{17}$

\section{What is the Role of Resilience?}

The historical term "hardiness" or the modern term one, "resilience" connotes the human propensity towards adaptability and those characteristics that allow clinicians to thrive despite adversity. ${ }^{18}$ These factors are equally important when considering burnout and its related conditions. Understanding protective factors is key to figuring out how to prevent and treat burnout and to provide effective coping mechanisms. While resilience tends to focus on individual factors, the culture of the work environment can certainly bolster resilient workers and teach many skills and habits that increase resilience (ie, resilience can be learned). For example, reducing stigma around mental health phenomena in clinicians is paramount along with providing adequate mental health services for clinicians and immediate debriefing strategies for tense or disturbing clinical situations (eg, Code Lavender). ${ }^{19}$ For physicians, state licensing boards should rethink questions pertaining to history of mental health disorders and treatments, which dissuade many physicians from seeking needed care. ${ }^{20}$

Resilience comes in many forms. The enhancement of clinician wellness should consider resilience attributes that were effective for clinicians at other times and why they may not work in a new or novel employment situation. In their research with prisoners of war, military personnel, holocaust survivors, and trauma survivors, Dennis Charney and Steven Southwick identified key protective factors associated with post-traumatic growth and resilience. Those resilience factors are the following: the confrontation of fear; maintaining an optimistic outlook; seeking and accepting social support; imitation of sturdy role models; relying on an inner moral compass; embracing spiritual or religious practice; accepting what cannot be changed; attending to health and wellbeing; creating meaning and opportunity from adversity; and accepting personal responsibility for emotional wellbeing. ${ }^{21}$ 


\section{Identifying and Addressing Psychiatric Morbidity}

In addition to a high prevalence of burnout and work-related mental health conditions, other non-work-related mental conditions such as depression, substance abuse and suicide are also higher in physicians and clinicians than in the public. $^{22}$ These mental health conditions in clinicians require their own types of mental health interventions. ${ }^{23}$ Unfortunately, clinicians often make heuristic assumptions, take short-cuts, or avoid contentious topics when they are treating other clinicians. It is with great irony that the healer ends up receiving compromised care that does not include standard screening for mental health conditions or properly done interviews on sensitive topics. ${ }^{24}$ Of course, clinicians are reluctant to seek care in the first place, which makes an accurate diagnosis even more important.

An effort should be made to understand the symptoms in relation to the work environment by asking how the clinician patient feels at work and away from work with a particular focus on thoughts about work, colleagues, and professional career trajectory while ascertaining how the clinician sense of self is affected independently of work. A detailed summary of functional impairment should be sought in relation to standard psychiatric conditions.

Work-related conditions such as burnout present similarly to depression but are inherently tied to the work environment and its conditions. ${ }^{25}$ Burnout results from a work-workplace mismatch whereas depression would not be expected to remit with changes in work environment. ${ }^{25}$ Both may present with decreased energy, apathy, listlessness, irritability, cynicism, inability to function (executive function), and a sense of lacking professional accomplishment. Both result in adverse consequences in patient care including worse clinical outcomes, poor patient satisfaction, and loss of revenue. Patients with depression may have more pronounced anhedonia, guilt, endorsement of depression, sense of worthlessness, hopelessness, anxiety, agitation, and suicidal ideations. ${ }^{26}$ Clearly, attention to serious mental health issues is critical to addressing treatment appropriately. Burnout and related conditions are expected to not reach the clinical threshold of Diagnostic Statistical Manual disorders, which would require different and more intensive treatments generally.

Therapeutic strategies to tackle burnout and emotional exhaustion should not only identify causative factors and root causes, but should clearly identify the work-related condition (eg, burnout, moral distress) while ruling out non-work-related mental health conditions.

\section{Interventions for Burnout and Related Conditions}

Generally, interventions can be divided into those that address the individual clinician or the organization or institution. Some interventions address both and some may be adaptable depending on the situation. It is also important to consider to measurement of the intended outcome. For example, many clinicians and researchers alter the use of the MBI, the most used burnout measure, for the domain of interest (eg, emotional exhaustion). Metaanalyses show that organization-based interventions not only induce a greater reduction in burnout but also exhibit a longer duration of response. ${ }^{27,28}$ In terms of burnout, even reduction of a few points on the MBI is clinically meaningful but the duration or benefit is also an important consideration. Among the individual-based interventions, those that incorporate mindfulness-based stress reduction or cognitive behavioral therapy tend to be most efficacious. $^{27}$ In general, drawing awareness into the clinical encounter and moment by moment interactions tends to reduce symptoms of burnout. Specific stresses of medical disciplines should also be considered in addition to general adaptation to stress.

It may be difficult for a clinician treating or experiencing burnout to decide whether to engage or disconnect from work, perhaps for a respite and internal reflection away from patient care. While disconnecting may be effective in the short term, learning ways to engage and cope with work stressors offers not only strategy but durability in terms of burnout prevention. Involvement in individual and collective spirituality practices can lead to greater compassion satisfaction and less burnout. Clinicians who are attentive to their own self- care tend to have higher levels of empathy and compassion towards patients. ${ }^{6}$ In terms of engaging with patients, palliative care offers some conceptual solutions. One in particular is the idea of "healing connections" posited by Dr. Balfour Mount, a pioneer in the palliative care field. ${ }^{29}$ It seems to resonate with patients and clinicians alike. Dr. Mount and his colleagues found that isolation and feeling disconnected were most associated with suffering and anguish. Many participants suffering from burnout and related conditions described an existential vacuum, a crisis of meaning, and an inability to find solace or inner peace. Patients express feelings of victimization and a need for 
control, which can be similarly expressed by burnt out clinicians. Ruminations about unsettling issues of the past and anxieties about the uncertain future consistently removed them from the potential of the present moment. Interestingly, these coping patterns frequently had their roots in early childhood, which speaks to how ingrained these patterns are and the necessary tenacity to fix change them. Therefore, the identification, cultivation, and perpetuation of healing connections benefits patients, families, and caregivers by providing meaning and purpose. These relationships may manifest themselves through subtle moments or ways and may be transient. It is important to not impart judgment in terms of what "should be" as this is most often tinged with the clinicians' ideals of the clinician-patient relationship, which may or may not be in line with what the patient is experiencing and her own assessment of the situation. Healing connections built on patients' needs are the most meaningful and effective.

Healing connections apply to the healers as well. Clinicians may work to cultivate those meaningful connections in their lives inside and outside of the work environment. ${ }^{30}$ Compassion practices, recognizing the need for and practicing self-compassion, and empathy enhancement can reduce burnout. ${ }^{14,31}$

\section{Interventions Targeting Individual Clinicians or Institution/ Organization}

This distinction is fundamentally important when one considers that burnout is a mismatch of worker to work-place. Therefore, it may not be surprising that organizational or structural changes tend to be more effective and for longer durations. Commonly, these systemic changes are straightforward such as duty hour restrictions for residents or coverage changes but may involve leadership changes or other more profound arrangements. ${ }^{27,28}$ Clinicians can feel blame (ie, stigma) for not doing enough on an individual level to prevent burnout. But attention at the individual level (eg, yoga or exercise practices, etc.) will have limited efficacy when the root cause of burnout is not addressed or in the setting of organizational dysfunction.

\section{Mindfulness-Based Stress Reduction and Other Mindfulness Practices}

Mindfulness-Based Stress Reduction (MBSR) is a protocolized intervention that teaches mindfulness and stress reduction techniques over approximately 8 weekly sessions. Its benefits are numerous as it enables perspective taking and enhances new ways of thinking and feeling about anxiety provoking situations. While many interventions may incorporate mindfulness techniques, MBSR is a specific form of mindfulness training that has the most robust data behind it in terms of burnout prevention and reduction to date. ${ }^{32}$ Attention to mindfulness, along with compassion, in the end-of-life setting was found to be effective in reducing clinician distress and enhancing clinician self-care. ${ }^{33}$

\section{Meaning-Based and Compassion-Based Interventions}

Meaning-Centered Therapy (MCP) is a protocolized prescriptive, time-limited group or individually based therapy that has proven efficacy for patients experiencing existential distress. It is based on the work of Victor Frankl, an Austrian psychoanalyst and holocaust survivor. It has been adapted for use in many disease-related settings and for clinicians. The restoration of meaning in clinicians working in palliative care settings is highly effective in reducing and preventing burnout. ${ }^{34}$

Joan Halifax developed two related models of compassion that are useful for developing and cultivating compassion. The first was the A.B.I.D.E. Model of Compassion. ${ }^{5}$ Attention and affect (A) refers to the ability to hold attention without distraction. Balance (B) refers to the mindfulness needed to perceive the reality of suffering without being overwhelmed by the magnitude or intensity of it. Intention refers to the drive or motivation to relieve suffering. Insight (I) refers to perceptual knowledge, which is integral to cultivate discernment (D) in difficult situations as part of a cognitive process. Embodiment, engagement, and equanimity (E) speak to the innate drive to connect with other people. Of note, intention (to alleviate suffering) is feature that differentiates compassion from empathy where the motivational or behavioral component is contested. Subsequently, Halifax developed the G.R.A.C. E. model as an active contemplative practice focused on cultivating compassion as we interact with others. ${ }^{5}$ G.R.A. C.E. is an applied model and stands for Gathering attention; Recalling intention; Attunement (to self and others); Consideration (open to insights and discernment of what will benefit the patient); and ethically Engaging/Enacting, and Ending the interaction. This process works to engender compassion as caregivers and fully engage in interactions with patients and families. 


\section{Implications for Frontline Medical Staff}

Medical staff working in emergency rooms, clinics, hospitals, or at the initial point of care, must contend with both acute and chronic stressors associated with burnout and related conditions. Therefore, immediate interventions that ameliorate the effects of difficult clinical situations on those frontline medical staff are instrumental and foundational to the mental health of these frontline workers. The same principles regarding institutional versus individually centered interventions still apply but certain interventions should happen in "real-time". Bor example, a "code lavender" program may be instituted in the hospital or department that allows for a multidisciplinary huddle that may be called or instituted by any clinical staff member who is in distress-The effect is to mobilize multi-disciplinary resources to implement a real-time check-in and debriefing of the situation. ${ }^{19}$ Mental health resources should be made readily available to frontline medical staff in distress that can also function as a point of care to triage the situation and provide a treatment plan. Frontline healthcare workers experience an abundance of pressure to provide appropriate and adequate care for their passions. Research demonstrates that efforts to facilitate workflow and enhance opportunities to provide compassionate care would reduce workplace stress and perhaps most importantly these potential changes (ie, its barriers and facilitators) were seen as doable and necessary. ${ }^{35}$

Redesigning workplace environments has received considerable attention as these structural forces seem to dictate a large percentage of burnout variance. ${ }^{36}$ Many of the ingredients for healthy user-friendly frontline medical environments are forthcoming. This could be everything from the physical space to use the EHR. ${ }^{37}$

\section{Effects of COVID-19 on the Emotional Well-Being of Front-Line Staff}

The effects of COVID-19 are unfolding on the backdrop of burnout and decrements to clinician well-being. There are unique features of pandemic-related changes to wellbeing. COVID-19 affects clinicians who are not on the front lines to a similar extent as those on the front lines treating COVID19 patients. ${ }^{13}$ This is qualitatively distinct from other sources of burnout in oncology where immediate work environment plays a prominent role. ${ }^{14}$ At the same time, locations where the pandemic has been mostly strongly active are also associated with higher rates of anxiety, distress, burnout and generally poor mental health among clinicians. ${ }^{15}$ PreCOVID-19 rates of burnout exacerbate the acute stressors caused by COVID-19 and the risk of post-traumatic stress disorder or other mental health outcomes among clinicians. ${ }^{16}$

\section{Summary}

Emotional exhaustion, burnout, empathic distress, and occupational stress are common and almost expectedly rampant among front-line medical stuff. The causes are myriad; many are consistent across frontline medical settings while others are unique to certain clinical environments. For example, mental health professionals who work on the front lines with acutely ill patients are prone to become victims of violence while emergency department staff are prone to burnout related to unpredictability. Long hours and limited control of the work schedule or environment are other consistent factors leading to burnout.

In general, understanding the root causes of burnout and related conditions is key to designing and implementing therapeutic interventions. ${ }^{38}$ Attention to issues of medical professional mental health has led to many important and timely interventions but their implementation and applicability across clinical settings has yet to be determined.

\section{Disclosure}

No conflict of interest reported by authors.

\section{References}

1. Freudenberger HJ. Staff burn-out. J Soc Issues. 1974;30(1):159-165. doi:10.1111/j.1540-4560.1974.tb00706.x

2. Maslach CJS. The Maslach Burnout Inventory. Palo Alto Consulting Psychologists Press; 1981.

3. Kristensen TSBM, Villadsen E, Christensen KB. The Copenhagen burnout inventory: a new tool for the assessment of burnout. Work Stress. 2005;1:152-207.

4. Montero-Marin J, Araya R, Blazquez BO, Skapinakis P, Vizcaino VM, Garcia-Campayo J. Understanding burnout according to individual differences: ongoing explanatory power evaluation of two models for measuring burnout types. BMC Public Health. 2012;12:922. doi:10.1186/1471-2458-12-922

5. Halifax J. Standing on the Edge: Finding Freedom Where Fear and Courage Meet. New York: Flatiron Books; 2018.

6. Vachon MLS, Huggard PK, Huggard JA. Reflections on occupational stress in pallaitive care nursing: is it changing? In: Ferrell CN, Paice J, editors. Textbook of Palliative Care Nursing. New Yor, NY: Oxford University Press; 2015.

7. Shanafelt TD, West CP, Sinsky C, et al. Changes in Burnout and Satisfaction With Work-Life Integration in Physicians and the General US Working Population Between 2011 and 2017. Mayo Clin Proc. 2019;94(9):1681-1694. doi:10.1016/j.mayocp.2018.10.023

8. Kamal AH, Bull JH, Wolf SP, et al. Prevalence and Predictors of Burnout Among Hospice and Palliative Care Clinicians in the U.S. $J$ Pain Symptom Manage. 2020;59(5):e6-e13. doi:10.1016/j. jpainsymman.2019.11.017 
9. Moukarzel A, Michelet P, Durand AC, et al. Burnout Syndrome among Emergency Department Staff: prevalence and Associated Factors. Biomed Res Int. 2019;2019:6462472. doi:10.1155/2019/ 6462472

10. Tawfik DS, Scheid A, Profit J, et al. Evidence Relating Health Care Provider Burnout and Quality of Care: a Systematic Review and Meta-analysis. Ann Intern Med. 2019;171(8):555-567. doi:10.7326/ M19-1152

11. Arnsten AFT, Shanafelt T. Physician Distress and Burnout: the Neurobiological Perspective. Mayo Clin Proc. 2021;96(3):763-769. doi:10.1016/j.mayocp.2020.12.027

12. Figley C. Compassion Fatigue, Coping with Secondary Traumatic Stress Disorder. New York: Brunner/Mazel; 1995.

13. Sinclair S, Raffin-Bouchal S, Venturato L, Mijovic-Kondejewski J, Smith-MacDonald L. Compassion fatigue: a meta-narrative review of the healthcare literature. Int J Nurs Stud. 2017;69:9-24. doi:10.1016/ j.ijnurstu.2017.01.003

14. Neville K, Cole DA. The relationships among health promotion behaviors, compassion fatigue, burnout, and compassion satisfaction in nurses practicing in a community medical center. $J$ Nurs Adm. 2013;43(6):348-354. doi:10.1097/NNA.0b013e3182942c23

15. Slocum-Gori S, Hemsworth D, Chan WW, Carson A, Kazanjian A. Understanding Compassion Satisfaction, Compassion Fatigue and Burnout: a survey of the hospice palliative care workforce. Palliat Med. 2013;27(2):172-178. doi:10.1177/0269216311431311

16. Jameton A. Dilemmas of moral distress: moral responsibility and nursing practice. AWHONNS Clin Issues Perinat Womens Health Nurs. 1993;4(4):542-551.

17. Walshchots M. Kant on Moral Satisfaction. Kantian Review. 2017;22 (2):281-303. doi:10.1017/S136941541700005X

18. Zwack J, Schweitzer J. If every fifth physician is affected by burnout, what about the other four? Resilience strategies of experienced physicians. Acad Med. 2013;88(3):382-389. doi:10.1097/ ACM.0b013e318281696b

19. Johnson B. Code Lavender: initiating holistic rapid response at the Cleveland Clinic. Beginnings. 2014;34(2):10-11.

20. Dyrbye LN, West CP, Sinsky CA, Goeders LE, Satele DV, Shanafelt TD. Medical Licensure Questions and Physician Reluctance to Seek Care for Mental Health Conditions. Mayo Clin Proc. 2017;92(10):1486-1493. doi:10.1016/j.mayocp.2017.06.020

21. Southwick SMC, Dennis S. Resilience: The Science of Mastering Life's Greatest Challenges. Cambridge University Press; 2012.

22. Schernhammer ES, Colditz GA. Suicide rates among physicians: a quantitative and gender assessment (meta-analysis). Am $J$ Psychiatry. 2004;161(12):2295-2302. doi:10.1176/appi. ajp.161.12.2295

23. Petrie K, Crawford J, Baker STE, et al. Interventions to reduce symptoms of common mental disorders and suicidal ideation in physicians: a systematic review and meta-analysis. Lancet Psychiatry. 2019;6(3):225-234. doi:10.1016/S2215-0366(18)30509-1

24. Montgomery AJ, Bradley C, Rochfort A, Panagopoulou E. A review of self-medication in physicians and medical students. Occup Med. 2011;61(7):490-497. doi:10.1093/occmed/kqr098

Psychology Research and Behavior Management

\section{Publish your work in this journal}

Psychology Research and Behavior Management is an international, peer-reviewed, open access journal focusing on the science of psychology and its application in behavior management to develop improved outcomes in the clinical, educational, sports and business arenas. Specific topics covered in the journal include: Neuroscience, memory and decision making; Behavior modification and management; Clinical
25. Wurm W, Vogel K, Holl A, et al. Depression-Burnout Overlap in Physicians. PLoS One. 2016;11(3):e0149913. doi:10.1371/journal. pone. 0149913

26. Epstein RM, Privitera MR. Physician burnout is better conceptualised as depression - Authors' reply. Lancet. 2017;389(10077):1398. doi:10.1016/S0140-6736(17)30898-X

27. West CP, Dyrbye LN, Erwin PJ, Shanafelt TD. Interventions to prevent and reduce physician burnout: a systematic review and meta-analysis. Lancet. 2016;388(10057):2272-2281. doi:10.1016/ S0140-6736(16)31279-X

28. Panagioti M, Panagopoulou E, Bower P, et al. Controlled Interventions to Reduce Burnout in Physicians: a Systematic Review and Meta-analysis. JAMA Intern Med. 2017;177 (2):195-205. doi:10.1001/jamainternmed.2016.7674

29. Mount BM, Boston PH, Cohen SR. Healing connections: on moving from suffering to a sense of well-being. J Pain Symptom Manage. 2007;33(4):372-388. doi:10.1016/j.jpainsymman.2006.09.014

30. Kearney M. The Nest in the Stream: Lessons from Nature on Being with Pain. Berkeley, CA Parralax Press; 2018.

31. Wilkinson H, Whittington R, Perry L, Eames C. Examining the relationship between burnout and empathy in healthcare professionals: a systematic review. Burn Res. 2017;6:18-29. doi:10.1016/ j.burn.2017.06.003

32. Lamothe M, Rondeau E, Malboeuf-Hurtubise C, Duval M, Sultan S. Outcomes of MBSR or MBSR-based interventions in health care providers: a systematic review with a focus on empathy and emotional competencies. Complement Ther Med. 2016;24:19-28. doi:10.1016/j.ctim.2015.11.001

33. Orellana-Rios CL, Radbruch L, Kern M, et al. Mindfulness and compassion-oriented practices at work reduce distress and enhance self-care of palliative care teams: a mixed-method evaluation of an "on the job" program. BMC Palliat Care. 2017;17(1):3. doi:10.1186/ s12904-017-0219-7

34. Fillion L, Vachon M, Gagnon P. Enhancing meaning at work and preventing burnout: the meaning-centered intervention for palliative care clinicians. Breitbart W, editor. Cambridge Oxford University Press; 2017. 168-181.

35. Singh P, Raffin-Bouchal S, McClement S, et al. Healthcare providers' perspectives on perceived barriers and facilitators of compassion: results from a grounded theory study. J Clin Nurs. 2018;27(910):2083-2097. doi:10.1111/jocn.14357

36. Wright AA, Katz IT. Beyond Burnout - Redesigning Care to Restore Meaning and Sanity for Physicians. $N$ Engl J Med. 2018;378 (4):309-311. doi:10.1056/NEJMp1716845

37. Nguyen OT, Jenkins NJ, Khanna N, et al. A systematic review of contributing factors of and solutions to electronic health record-related impacts on physician well-being. $J$ Am Med Inform Assoc. 2021;28(5):974-984. doi:10.1093/jamia/ocaa339

38. Squiers JJ, Lobdell KW, Fann JI, DiMaio JM. Physician Burnout: are We Treating the Symptoms Instead of the Disease? Ann Thorac Surg. 2017;104(4):1117-1122. doi:10.1016/j.athoracsur.2017.08.009 applications; Business and sports performance management; Socia and developmental studies; Animal studies. The manuscript management system is completely online and includes a very quick and fair peer-review system, which is all easy to use. Visit http://www. dovepress.com/testimonials.php to read real quotes from published authors. 